\title{
A comparative study of the antimicrobial activity of oregano, rosemary and thyme essential oils against Salmonella spp
}

\author{
Grammato Evangelopoulou $^{1 *}$, Nikolaos Solomakos ${ }^{2}$, Anastasios Ioannidis ${ }^{1}$, Andreana Pexara ${ }^{2}$ and Angeliki R Burriel $^{1}$ \\ ${ }^{1}$ Department of Nursing, University of Peloponnese, Sparta, Greece \\ ${ }^{2}$ Laboratory of Hygiene of Foods of Animal Origin, Faculty of Veterinary Medicine, University of Thessaly, 224 Trikalon Street, 43100 Karditsa, Greece
}

\begin{abstract}
The emergence of Salmonella resistance to therapeutic agents against human infections is attributed to their excessive use in the treatment of food-producing animals. The spreading of resistance necessitates vigorous research on alternative therapeutic schemes in order to replace commonly used antimicrobials. Thus, the aim of the present study was to investigate the inhibitory activity of three commercially available essential oils (EOs) against fifty-nine Salmonella strains belonging to fourteen serovars isolated from pig carcasses at slaughter. Their antimicrobial activity was compared with that of twenty-four commonly used antimicrobials previously tested against the same strains. The three chosen EOs were oregano, rosemary and thyme. Oregano and thyme EOs showed high antimicrobial activity, compared to that of rosemary and a better antimicrobial effect compared to that of commercial antimicrobials. The results showed that oregano and thyme have the potential of an effective alternative treatment against Salmonella spp., especially those strains resistant to synthetic antimicrobials. Thus, some EOs could be a safer alternative treatment against certain infections of food-producing animals or, perhaps, growth promoters, used for effective prevention.
\end{abstract}

\section{Introduction}

Food-producing animals are a widely recognized source for Salmonella species causing human infections. Pork meat and products are major sources of foodborne salmonellosis in the European Union (EU) and around the world. Subclinically infected pigs, incriminated for carcass contamination at slaughter, are causing 10 to $20 \%$ of human cases of salmonellosis in the EU [1,2].

The most important threat to human health is the emergence of multidrug resistant Salmonella strains, mainly belonging to serovar Typhimurium. Pigs are recognized as a primary reservoir of such strains, necessitating control measures for public health protection [3-7]. Unregulated use of antibiotics in farming practices for many decades, causing selective pressure, is thought the main reason for this emergence [8-12]. The amounts of antimicrobials used and the ways those drugs are administrated affect the resistance of gut microflora [9]. Therapeutics, such as ampicillin, trimethoprim-sulfamethoxazole, third generation cephalosporins and fluoroquinolones, effective for treating human Salmonella infection, are also administered to food producing animals, implicated in the transmission of resistant strains $[13,14]$. Thus, the spreading of multi-resistant Salmonella through mobile genetic elements has become a global threat to effectively treating human cases, particularly in immunocompromised individuals with increased susceptibility to accidental Salmonella infection $[15,16]$.

The increasing prevalence of antibiotic resistant bacterial strains promotes the investigation for new antimicrobial methods in order to control infections in animals. Among them is the application of plant essential oils (EOs) against a wide range of microorganisms [17]. EOs, also called volatile or ethereal oils, are aromatic oily plant liquid extracts recognized for their antimicrobial properties since ancient times and having recently re-emerged as safe, natural antimicrobials
[18-22]. Research has revealed that the EOs' high content of phenolic derivatives, such as carvacrol and thymol, target the bacterial membrane transport system, causing disruption at the cytoplasmic homeostasis, affecting finally cell respiration and the microbial enzyme system [2325]. These properties of medicinal interest, in evidence of dangerous increases of microbial resistance to conventional treatments [26], has increased hope that EOs could become safe to public health when used as potential natural growth promoters (NGPs) for farmed animals. Specifically, their inclusion in animal diets for replacing antibiotics used as growth promoters (AGPs), could improve quality of gut microbiota thus, growth performance of animals and animal welfare [27], eventually contributing to consumer safety.

The disinfectant and antibacterial properties of oregano EO were first recognized in ancient Greece, where it was often used for treating bacterial skin and wound infections. It was also used to protect food from the growth of bacteria. Oregano (Origanum vulgare subsp. hirtum, a herb of the Labiatae family) is a plant that strives on mountainous terrain, from where it got its name meaning 'Delight of the Mountains' [28,29]. Carvacrol and thymol are the two main phenols, constituting about $78.85 \%$ of oregano EOs and responsible for the plant's antimicrobial activity [30]. Carvacrol in particular, has been

*Correspondence to: Grammato Evangelopoulou, Department of Nursing, Faculty of Human Movement and Quality of Life Sciences, Laboratory of Integrated Healthcare, University of Peloponnese, Sparta, Greece, Tel: 30-694584-7840, E-mail: matinavet@hotmail.com

Key words: antimicrobial resistance, essential oil, pig, Salmonella

Received: December 06, 2019; Accepted: December 20, 2019; Published: December 23, 2019 
found by many as possessing remarkable antimicrobial properties [3134]. In addition, other minor constituents, such as the monoterpene hydrocarbons g-terpinene and pcymene are also reported as contributors to the antibacterial activity of the oil [35]. There are, also, reports on the chemical composition and the antimicrobial properties of the EOs of various oregano species, and their application in different commercial preparations, as antimicrobials and antioxidants $[32,36]$.

Rosemary (Rosmarinus officinalis) or 'dew of the sea' (Latin ros for 'dew' and for 'sea'), is a member of the mint family Lamiaceae [37]. Its major constituents are 1,8 -Cineole $(26.54 \%)$ and $\alpha$-Pinene (20.14\%) [38]. This fragrant evergreen herb has been known since ancient times for its medicinal properties. It was traditionally used to boost the immune system [39]. Among the pharmacologically validated medicinal uses of rosemary are anticancer, antidiabetic, anti-inflammatory and antinociceptive, antioxidant, antithrombotic, antiulcerogenic, improving cognitive deficits, antidiuretic, hepatoprotective and antibacterial effects against Gram positive and negative microorganisms $[38,40,41]$.

Thyme belongs to the genus Thymus of the mint family (Lamiaceae) and is a relative of the genus Origanum. Its EO contains more than 60 ingredients, most of which possess important antioxidant and antimicrobial properties [42]. The essential oil of common thyme (Thymus vulgaris) contains $20-54 \%$ thymol, which is the compound [43] primarily responsible for its antioxidative activity, including Salmonella spp. [44], but similar activity have also carvacrol (2.2$4.2 \%)$, the monoterpene hydrocarbons p-cymene (18.5-23.5\%) and g-terpinene (16.1-18.9\%) [29,45]. These chosen to be investigated EOs are native to temperate Western and Southwestern Eurasia and especially the Mediterranean region [46] including Greece [47], with climatic conditions and geographical morphology promoting the growth of many species of aromatic plants. The antibacterial activity of oregano, rosemary and thyme has been studied against various multidrug resistant pathogenic bacteria, including foodborne pathogens, such as Salmonella spp. and E.coli $[29,48]$.

Therefore, the objective of the present study was primarily to determine the activity of oregano, rosemary and thyme against 59 pig Salmonella spp. isolates and also compare it to the known antimicrobial activities of commonly used antimicrobials against the same isolates. The study focused on i) the investigation of the EOs' inhibitory effect in relation to the amount used ii) the comparison of the effectiveness of each EO used and iii) the investigation of each EO's bactericidal effect on the selected serovars as compared to commercial antimicrobials.

\section{Materials and methods}

\section{Isolation, serotyping and antimicrobial susceptibility testing of Salmonella spp.}

Fifty-nine (59) Salmonella strains, isolated from 615 pig tissue samples (ileum, mesenteric lymph nodes, faecal samples, gallbladder and neck muscle) collected from 123 pigs were investigated as described elsewhere [49-51].

The isolates belonged to fourteen serovars, which were 21 (35.6\%) Salmonella Typhimurium, 9 (15.25\%) S. enterica subsp. enterica ser. 4,12:i:-, 7 (11.86\%) S. enterica subsp. enterica ser. 6,7:k:-, 6 (10.17\%), S. enterica subsp. enterica ser. 4,5, 12:i:-, 3 (5.1\%), S. Bredeney and from one isolate each (1.7\%) for $S$. Agona, $S$. Derby, $S$. Infantis, $S$. Meleagridis, S. Cerro, S.enterica subsp. enterica ser. 6,14,25:-:1,2, S. enterica subsp. diarizonae 61:k:1,5, S. enterica subsp. salamae 38:b:1,2, S. enterica subsp. houtenae 40:g,t:-, while 4 (6.8\%) were characterized as 'Rough'.
The same isolates had been previously tested against 24 antimicrobials, routinely used for animal and human treatments $[49,50]$. The commercially available (Thermo Scientific ${ }^{\mathrm{mm}}$ Oxoid $^{\mathrm{ms}}$ ) antimicrobial susceptibility disks used were amoxicillin (30 $\mu \mathrm{g})$, amoxicillin/ clavulanic acid $(20 / 10 \mu \mathrm{g})$, ampicillin $(10 \mu \mathrm{g})$, ampicillin/sulbactam $(10 / 10 \mu \mathrm{g})$, aztreonam $(30 \mu \mathrm{g})$, cefotaxime $(30 \mu \mathrm{g})$, cefoxitin $(30 \mu \mathrm{g})$, ceftazidime $(30 \mu \mathrm{g})$, ceftiofur $(30 \mu \mathrm{g})$, ceftriaxone $(30 \mu \mathrm{g})$, cefuroxime $(30 \mu \mathrm{g})$, chloramphenicol $(30 \mu \mathrm{g})$, colistin $(50 \mu \mathrm{g})$, doripenem $(10$ $\mu \mathrm{g})$, enrofloxacin $(5 \mu \mathrm{g})$, erythromycin $(15 \mu \mathrm{g})$, gentamycin $(10 \mu \mathrm{g})$, kanamycin $(30 \mu \mathrm{g})$, nalidixic acid $(30 \mu \mathrm{g})$, penicillin $\mathrm{G}(10 \mu \mathrm{g})$, rifampin $(30 \mu \mathrm{g})$, sulfamethoxazole/trimethoprim $(23.75 / 1.25 \mu \mathrm{g})$, tetracycline $(30 \mu \mathrm{g})$ and tigecycline $15(\mu \mathrm{g})$.

\section{Antimicrobial activity of EOs}

The disk diffusion method was applied for the determination of the antibacterial activity of the three selected EOs: oregano, rosemary and thyme (ECO PHARM, Greece, http://www.ecopharm.gr/index.php/ products.html). The inhibitory effect of each EO was examined against three graded undiluted commercial EOs, according to Skandamis et al. [52]. Each bacterial culture was standardized to the range of $0.5 \mathrm{Mc}$ Farland standard and inoculated onto Mueller-Hinton agar (LMLAB 39). Sterilized Whatman paper discs $6 \mathrm{~mm}$ in diameter (Difco, Becton, Dickinson, Sparks, MD) were impregnated with 5, 15 and $30 \mu$ of the EOs. Disks impregnated in absolute alcohol were used as negative controls. Disks with EOs and the negative control were placed on the surface of petri plates inoculated with Salmonella. The cultures were incubated at $37^{\circ} \mathrm{C}$ for $18-24 \mathrm{~h}$ and the zones of inhibition were measured and recorded in the same way as for commercial antimicrobials. Specifically, bacterial growth inhibition was the diameter of the zones $(\mathrm{mm})$ without bacterial growth. For the interpretation of the results, four groups of inhibitory zones were examined: i) no inhibition, ii) $<12 \mathrm{~mm}$ (weak activity zone), iii) $12 \mathrm{~mm} \geq$ inhibition zone $<20 \mathrm{~mm}$ (intermediate activity) and iv) inhibition zone $\geq 20 \mathrm{~mm}$ (strong activity) [53].

\section{Statistical analysis}

The Statistical Package for Social Sciences (SPSS) version 17 was used for the statistical analysis of results. Statistical significance was set as $\mathrm{p}<0.05$ or $\mathrm{p}<0.001$.

Quantitative data were presented as mean and standard deviation and qualitative as percentage. The normality assumption was tested with the Shapiro Wilk test, since the size of each group was small [54]. To compare the mean between quantitative variables the MannWhitney, T-test and one-way ANOVA were used [55].

\section{Results}

\section{Antimicrobial susceptibility testing}

The previously reported resistance of the examined isolates $[49,50]$ against commercially used antimicrobials is re-reported with table 1 for a comparison with the findings of each EO's activity. As has been reported the highest proportions of resistance were observed for penicillin $\mathrm{G}$, rifampin, tetracycline, amoxicillin, ampicillin and sulfamethoxazole/ trimethoprim (52-88\%). Medium resistance was observed for kanamycin, chloramphenicol and colistin. Twenty- six isolates (44\%) displayed resistance to at least three antimicrobial categories, while all Salmonella isolates were susceptible to cephalosporins, quinolones, doripenem and aztreonam.

\section{Antimicrobial activity of EOs}

The in vitro antibacterial activity of EOs, against the Salmonella isolates, was qualitatively assessed by the presence or absence of 
Table 1. Cumulative results of antimicrobial resistance of Salmonella spp. isolates recovered from pigs.

\begin{tabular}{|c|c|c|c|c|c|c|c|c|c|c|c|c|c|c|c|c|}
\hline 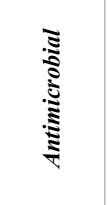 & 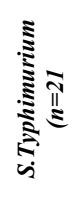 & 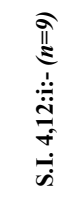 & 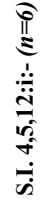 & 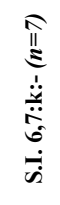 & 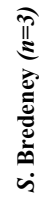 & 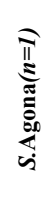 & 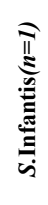 & 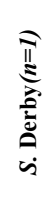 & 的弯 & 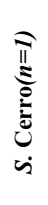 & 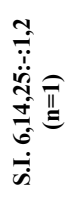 & 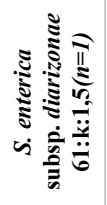 & 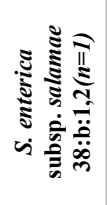 & 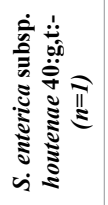 & 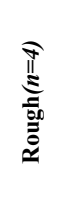 & 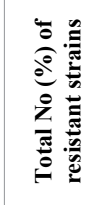 \\
\hline AML & 12 & 8 & 6 & 1 & 0 & 0 & 0 & 0 & 0 & 0 & 1 & 0 & 0 & 0 & 3 & $31(52.54)$ \\
\hline AMC & 0 & 0 & 0 & 0 & 0 & 0 & 0 & 0 & 0 & 0 & 0 & 0 & 0 & 0 & 1(I) & 0 \\
\hline AMP & 12 & 8 & 6 & 1 & 0 & 0 & 0 & 0 & 0 & 0 & 1 & 0 & 0 & 0 & 3 & $31(52.54)$ \\
\hline SAM & 1(I) & 1 & 1 & 0 & 0 & 0 & 0 & 0 & 0 & 0 & 0 & 0 & 0 & 0 & 0 & $2(3.4)$ \\
\hline AZT & 0 & 0 & 0 & 0 & 0 & 0 & 0 & 0 & 0 & 0 & 0 & 0 & 0 & 0 & 0 & 0 \\
\hline CTX & 0 & 0 & 0 & 0 & 0 & 0 & 0 & 0 & 0 & 0 & 0 & 0 & 0 & 0 & 0 & 0 \\
\hline FOX & 0 & $1(\mathrm{I})$ & 0 & 0 & 0 & 0 & 0 & 0 & 0 & 0 & 0 & 0 & 0 & 0 & 0 & 0 \\
\hline CAZ & 0 & 0 & 0 & 0 & 0 & 0 & 0 & 0 & 0 & 0 & 0 & 0 & 0 & 0 & 0 & 0 \\
\hline EFT & 0 & 0 & 0 & 0 & 0 & 0 & 0 & 0 & 0 & 0 & 0 & 0 & 0 & 0 & 0 & 0 \\
\hline CRO & 0 & 0 & 0 & 0 & 0 & 0 & 0 & 0 & 0 & 0 & 0 & 0 & 0 & 0 & 0 & 0 \\
\hline CXM & 4(I) & 0 & 0 & 0 & 0 & 0 & 0 & 0 & 0 & 0 & 0 & 0 & 0 & 0 & 0 & 0 \\
\hline $\mathrm{CT}$ & 2 & 2 & 0 & 1 & 0 & 0 & 0 & 0 & 0 & 1 & 0 & 1 & 0 & 0 & 0 & $7(11.86)$ \\
\hline C & 4 & 1 & 1 & 1 & 0 & 0 & 0 & 0 & 0 & 0 & 1 & 0 & 1 & 1 & 3 & $13(22)$ \\
\hline DOR & 0 & 0 & 0 & 0 & 0 & 0 & 0 & 0 & 0 & 0 & 0 & 0 & 0 & 0 & 0 & 0 \\
\hline ENR & 0 & 0 & 0 & 0 & 0 & 0 & 0 & 0 & 0 & 0 & 0 & 0 & 0 & 0 & 0 & 0 \\
\hline E & 21 & 9 & 6 & 7 & 3 & 1 & 1 & 1 & 1 & 1 & 1 & 1 & 1 & 1 & 4 & $59(100)$ \\
\hline $\mathrm{CN}$ & 1 & 2 & 1 & 0 & 0 & 0 & 0 & 0 & 0 & 0 & 0 & 0 & 0 & 0 & 0 & $4(8.1)$ \\
\hline $\mathrm{K}$ & 4 & 1 & 5 & 1(I) & 3 & 0 & 0 & 0 & 0 & 0 & 0 & 0 & 1(I) & 1(I) & 1 & $14(23.73)$ \\
\hline NA & 0 & 0 & 0 & 0 & 0 & 0 & 0 & 0 & 0 & 0 & 0 & 0 & 0 & 0 & 0 & 0 \\
\hline $\mathrm{P}$ & 18 & 9 & 6 & 6 & 2 & 1 & 1 & 1 & 0 & 1 & 1 & 0 & 1 & 1 & 4 & $52(88.13)$ \\
\hline $\mathrm{RD}$ & $\begin{array}{l}\text { 15, } \\
6(\mathrm{I})\end{array}$ & $7,2(\mathrm{I})$ & 6 & $\begin{array}{c}6, \\
1(\mathrm{I})\end{array}$ & 3 & 1 & 1 & 1 & 1 & $\begin{array}{l}1, \\
\text { (I) }\end{array}$ & 1 & $\begin{array}{l}\text { 1, } \\
\text { (I) }\end{array}$ & 0 & 1 & 4 & $49(83)$ \\
\hline SXT & 10 & 7 & 6 & 1 & 0 & 0 & 0 & 0 & 0 & 0 & 1 & 0 & 1 & 1 & 4 & $31(52.54)$ \\
\hline $\mathrm{TE}$ & 11 & 9 & 6 & 1 & 3 & 0 & 0 & 0 & 0 & 0 & 1 & 0 & 1 & 1 & 4 & $37(62.71)$ \\
\hline $\mathrm{TGC}$ & 0 & 0 & 0 & 0 & 0 & 0 & 0 & 0 & 0 & 0 & 1(I) & 0 & 0 & 1(I) & 0 & 0 \\
\hline Total N. & 21 & 9 & 6 & 7 & 3 & 1 & 1 & 1 & 1 & 1 & 1 & 1 & 1 & 1 & 4 & 59 \\
\hline
\end{tabular}

AML: Amoxicillin; $A M C$ : Amoxicillin-Clavulanic Acid; $A M P$ : Ampicillin; SAM: Ampicillin-Sulbactam; $A Z T$ : Aztreonam; $C T X:$ Cefotaxime; FOX: Cefoxitin; $C A Z$ : Ceftazidime; EFT: Ceftiofur; CRO: Ceftriaxone; CXM: Cefuroxime; $C$ : Chloramphenicol; $C T$ : Colistin; ENR: Enrofloxacin; E: erythromycin; $C N$ : Gentamycin; $K$ : Kanamycin; $N A$ : Nalidixic Acid; $P$ : Penicillin; G: RD Rifampin; SXT: Sulfamethoxazole/Trimethoprim; TE: Tetracycline; TGC: Tigecycline; I: Intermediate Resistance.

inhibition zones and quantitatively by the zone diameters. The zones of inhibition for each of the 3 amounts of the EOs are presented in table 2.

Oregano and thyme gave in all tested concentrations $(5,15$ and $30 \mu \mathrm{l})$ large inhibitory zones ranging from 20 to $60 \mathrm{~mm}$ for oregano and 11-70 $\mathrm{mm}$ for thyme, while rosemary exhibited smaller inhibition zones, or it did not show any activity. The inhibition zones ranged from 10 to $50 \mathrm{~mm}$ (table 3). The negative control (absolute alcohol) did not inhibit growth for any of the tested strains of Salmonella spp.

\section{Statistical analysis}

The findings are present in tables 3 and 4. All EOs showed a statistical significance between the Zone of Inhibition (ZOI) and the tested concentrations of 5-15, 5-30 and 15-30 $\mu \mathrm{l}(\mathrm{F}=240,95, \mathrm{p}<0,001$ for oregano, $F=188,592, p<0,001$ for thyme and $F=15,79, p<0,001$ for rosemary). Specifically:

i) oregano: mean $\mathrm{ZOI}$ for: $5-15 \mu \mathrm{l}(\mathrm{t}=-12.660, \mathrm{df}=58,2$-tailed $\mathrm{p}<0.001), 5-30 \mu \mathrm{l}(\mathrm{t}=-18.828, \mathrm{df}=58,2$-tailed $\mathrm{p}<0.001)$ and $15-30 \mu \mathrm{l}(\mathrm{t}=-$ 11.502, $\mathrm{df}=58,2$-tailed $\mathrm{p}<0.001)$.

ii) thyme: mean ZOI for: $5-15 \mu \mathrm{l}(\mathrm{t}=-9.389, \mathrm{df}=58,2$-tailed $\mathrm{p}<0.001), 5-30 \mu \mathrm{l}(\mathrm{t}=-8.685, \mathrm{df}=58,2$-tailed $\mathrm{p}<0.001)$ and $15-30 \mu \mathrm{l}(\mathrm{t}=-$ 10.385, df $=58,2$-tailed $\mathrm{p}<0.001)$.

iii) rosemary: mean $\mathrm{ZOI}$ for: $5-15 \mu \mathrm{l}(\mathrm{t}=-3.791, \mathrm{df}=58,2$-tailed $\mathrm{p}<0.001), 5-30 \mu \mathrm{l}(\mathrm{t}=-4.201, \mathrm{df}=58,2$-tailed $\mathrm{p}<0.001)$ and $15-30 \mu \mathrm{l}(\mathrm{t}=-$ 3.397, $\mathrm{df}=58,2$-tailed $\mathrm{p}<0.001)($ Tables 3,4$)$.
Repeated Measures ANOVA showed that the kind of the EO employed, affects significantly the width of ZOI $(\mathrm{F}=457,8, \mathrm{p}<0,001)$ (Table 5). Analytically, the mean ZOI of 5 and $15 \mu$ differed significantly between oregano and rosemary $(\mathrm{t}=40,558, \mathrm{df}=58,2$-tailed $\mathrm{p}<0,001)$ and between rosemary and thyme $(\mathrm{t}=-24.612, \mathrm{df}=58,2$-tailed $\mathrm{p}<0.001)$. No significance was observed between oregano and thyme $(\mathrm{t}=-0.883, \mathrm{df}=58$, 2 -tailed $\mathrm{p}=0.381)$. However, a statistical significance was observed for the ZOI among the three pairs of the EOs (95\% CI) at the concentrate of $30 \mu \mathrm{l}$.

Analysis of one-way variance showed a statistical significance among Salmonella serovars and the effect of oregano, rosemary and thyme (Sig. $=0.009$, Sig. $<0.001$, Sig. $<0.001$, respectively).

\section{Discussion and conclusion}

The use of antimicrobials, such as penicillin, tetracycline and their newer compounds is critical in guarding human and animal health [56]. However, their excessive use in veterinary medicine during the past decades has led, due to selection pressures, to the emergence of antibiotic-resistant bacteria transmitted to humans through the food chain [57].

The reported proportion $[13,14,58-60]$ of Salmonella resistance to tetracycline, amoxicillin, ampicillin, sulfamethoxazole/trimethoprim and chloramphenicol, antimicrobials considered as Highly Important for Human Health [61], is reflecting the extensive use of such agents 
Table 2. Inhibition zones (measured as $\mathrm{mm}$ ) of the Essential Oils at different concentrations against Salmonella spp. isolates.

\begin{tabular}{|c|c|c|c|c|c|c|c|c|c|}
\hline \multirow[b]{2}{*}{ SEROVAR } & \multicolumn{3}{|c|}{ OREGANO } & \multicolumn{3}{|c|}{ ROSEMARY } & \multicolumn{3}{|c|}{ THYME } \\
\hline & $5 \mu \mathrm{l}$ & $15 \mu \mathrm{l}$ & $30 \mu \mathrm{l}$ & $5 \mu \mathrm{I}$ & $15 \mu \mathrm{I}$ & $30 \mu \mathrm{I}$ & $5 \mu \mathrm{l}$ & $15 \mu \mathrm{I}$ & $30 \mu \mathrm{l}$ \\
\hline S. Typhimurium & 30 & 40 & 44 & $\mathrm{R}$ & $\mathrm{R}$ & $\mathrm{R}$ & 20 & 21 & 35 \\
\hline S. Typhimurium & 30 & 42 & 43 & $\mathrm{R}$ & $\mathrm{R}$ & $\mathrm{R}$ & 20 & 28 & 31 \\
\hline S. Typhimurium & 30 & 40 & 40 & $\mathrm{R}$ & $\mathrm{R}$ & $\mathrm{R}$ & 20 & 25 & 32 \\
\hline S. Typhimurium & 30 & 32 & 35 & $\mathrm{R}$ & $\mathrm{R}$ & $\mathrm{R}$ & 20 & 35 & 48 \\
\hline S. Typhimurium & 27 & 30 & 35 & $\mathrm{R}$ & $\mathrm{R}$ & $\mathrm{R}$ & 42 & 45 & 58 \\
\hline S. Typhimurium & 26 & 32 & 40 & $\mathrm{R}$ & $\mathrm{R}$ & $\mathrm{R}$ & 40 & 42 & 60 \\
\hline S. Typhimurium & 25 & 30 & 35 & $\mathrm{R}$ & $\mathrm{R}$ & $\mathrm{R}$ & 30 & 42 & 47 \\
\hline S. Typhimurium & 25 & 32 & 42 & $\mathrm{R}$ & $\mathrm{R}$ & $\mathrm{R}$ & 40 & 41 & 60 \\
\hline S. Typhimurium & 28 & 33 & 45 & $\mathrm{R}$ & $\mathrm{R}$ & $\mathrm{R}$ & 40 & 42 & 60 \\
\hline S. Typhimurium & 30 & 32 & 35 & $\mathrm{R}$ & $\mathrm{R}$ & $\mathrm{R}$ & 20 & 40 & 50 \\
\hline S. Typhimurium & 30 & 32 & 41 & $\mathrm{R}$ & 10 & 11 & 20 & 28 & 31 \\
\hline S. Typhimurium & 33 & 34 & 40 & $\mathrm{R}$ & 11 & 13 & 25 & 33 & 36 \\
\hline S. Typhimurium & 28 & 35 & 35 & $\mathrm{R}$ & $\mathrm{R}$ & $\mathrm{R}$ & 16 & 34 & 35 \\
\hline S. Typhimurium & 27 & 40 & 40 & $\mathrm{R}$ & $\mathrm{R}$ & 15 & 35 & 50 & 50 \\
\hline S. Typhimurium & 21 & 23 & 37 & $\mathrm{R}$ & $\mathrm{R}$ & $\mathrm{R}$ & 21 & 40 & 51 \\
\hline S. Typhimurium & 27 & 37 & 50 & $\mathrm{R}$ & $\mathrm{R}$ & $\mathrm{R}$ & 22 & 22 & 60 \\
\hline S. Typhimurium & 25 & 31 & 35 & $\mathrm{R}$ & $\mathrm{R}$ & $\mathrm{R}$ & 16 & 26 & 34 \\
\hline S. Typhimurium & 22 & 30 & 40 & $\mathrm{R}$ & $\mathrm{R}$ & 12 & 11 & 12 & 37 \\
\hline S. Typhimurium & 36 & 38 & 37 & $\mathrm{R}$ & $\mathrm{R}$ & $\mathrm{R}$ & 22 & 23 & 32 \\
\hline S. Typhimurium & 22 & 31 & 41 & $\mathrm{R}$ & 11 & 11 & 24 & 33 & 44 \\
\hline S. Typhimurium & 25 & 30 & 35 & $\mathrm{R}$ & $\mathrm{R}$ & $\mathrm{R}$ & 30 & 41 & 46 \\
\hline S.I. 4,12:i:- & 24 & 36 & 40 & $\mathrm{R}$ & $\mathrm{R}$ & $\mathrm{R}$ & 28 & 38 & 40 \\
\hline S.I. 4,12:i:- & 30 & 45 & 53 & $\mathrm{R}$ & $\mathrm{R}$ & $\mathrm{R}$ & 38 & 47 & 55 \\
\hline S.I. 4,12:i:- & 30 & 42 & 56 & $\mathrm{R}$ & $\mathrm{R}$ & $\mathrm{R}$ & 35 & 43 & 55 \\
\hline S.I. 4,12:i:- & 30 & 50 & 60 & $\mathrm{R}$ & $\mathrm{R}$ & $\mathrm{R}$ & 35 & 45 & 60 \\
\hline S.I. 4,12:i:- & 32 & 33 & 43 & $\mathrm{R}$ & $\mathrm{R}$ & $\mathrm{R}$ & 40 & 43 & 63 \\
\hline S.I. 4,12:i:- & 24 & 30 & 40 & $\mathrm{R}$ & $\mathrm{R}$ & $\mathrm{R}$ & 20 & 28 & 30 \\
\hline S.I. 4,12:i:- & 21 & 30 & 38 & $\mathrm{R}$ & $\mathrm{R}$ & $\mathrm{R}$ & 30 & 32 & 40 \\
\hline S.I. 4,12:i:- & 25 & 27 & 41 & $\mathrm{R}$ & 10 & 11 & 24 & 40 & 40 \\
\hline S.I. 4,12:i:- & 20 & 30 & 30 & $\mathrm{R}$ & $\mathrm{R}$ & $\mathrm{R}$ & 40 & 40 & 40 \\
\hline S.I. 4,5,12:i:- & 40 & 42 & 46 & $\mathrm{R}$ & $\mathrm{R}$ & $\mathrm{R}$ & 40 & 44 & 47 \\
\hline S.I. 4,5,12:i:- & 30 & 40 & 44 & $\mathrm{R}$ & $\mathrm{R}$ & $\mathrm{R}$ & 20 & 22 & 30 \\
\hline S.I. 4,5,12:i:- & 30 & 30 & 45 & $\mathrm{R}$ & 10 & 15 & 28 & 32 & 42 \\
\hline S.I. $4,5,12: i:-$ & 25 & 38 & 40 & $\mathrm{R}$ & 10 & 10 & 20 & 21 & 35 \\
\hline S.I. 4,5,12:i:- & 27 & 32 & 45 & $\mathrm{R}$ & $\mathrm{R}$ & $\mathrm{R}$ & 25 & 42 & 45 \\
\hline S.I. 4,5,12:i:- & 29 & 30 & 34 & $\mathrm{R}$ & $\mathrm{R}$ & 10 & 20 & 22 & 30 \\
\hline S.I. 6,7:k:- & 22 & 31 & 35 & $\mathrm{R}$ & $\mathrm{R}$ & $\mathrm{R}$ & 20 & 40 & 50 \\
\hline S.I. 6,7:k:- & 22 & 31 & 35 & $\mathrm{R}$ & $\mathrm{R}$ & $\mathrm{R}$ & 20 & 40 & 50 \\
\hline S.I. 6,7:k:- & 20 & 32 & 34 & $\mathrm{R}$ & $\mathrm{R}$ & $\mathrm{R}$ & 22 & 41 & 52 \\
\hline S.I. 6,7:k:- & 22 & 31 & 41 & $\mathrm{R}$ & 12 & 12 & 25 & 35 & 40 \\
\hline S.I. 6,7:k:- & 20 & 31 & 34 & $\mathrm{R}$ & $\mathrm{R}$ & $\mathrm{R}$ & 22 & 41 & 50 \\
\hline S.I. 6,7:k:- & 21 & 31 & 35 & $\mathrm{R}$ & $\mathrm{R}$ & $\mathrm{R}$ & 20 & 40 & 50 \\
\hline S.I. 6,7:k:- & 22 & 32 & 38 & $\mathrm{R}$ & $\mathrm{R}$ & $\mathrm{R}$ & 40 & 45 & 58 \\
\hline S. Bredeney & 21 & 30 & 40 & $\mathrm{R}$ & $\mathrm{R}$ & $\mathrm{R}$ & 40 & 42 & 65 \\
\hline S. Bredeney & 21 & 29 & 42 & $\mathrm{R}$ & $\mathrm{R}$ & $\mathrm{R}$ & 40 & 41 & 70 \\
\hline S. Bredeney & 30 & 32 & 35 & $\mathrm{R}$ & $\mathrm{R}$ & $\mathrm{R}$ & 20 & 40 & 50 \\
\hline S.Agona & 31 & 45 & 47 & $\mathrm{R}$ & $\mathrm{R}$ & $\mathrm{R}$ & 25 & 30 & 40 \\
\hline S.Infantis & 27 & 33 & 43 & $\mathrm{R}$ & $\mathrm{R}$ & $\mathrm{R}$ & 35 & 50 & 50 \\
\hline S.Derby & 27 & 40 & 40 & $\mathrm{R}$ & $\mathrm{R}$ & $\mathrm{R}$ & 25 & 42 & 45 \\
\hline S.Meleagridis & 38 & 47 & 55 & $\mathrm{R}$ & $\mathrm{R}$ & $\mathrm{R}$ & 38 & 48 & 60 \\
\hline S.Cerro & 25 & 30 & 35 & $\mathrm{R}$ & $\mathrm{R}$ & $\mathrm{R}$ & 30 & 33 & 40 \\
\hline S. enterica subsp. diarizonae $61: \mathrm{k}: 1,5$ & 38 & 48 & 55 & 13 & 21 & 28 & 38 & 45 & 60 \\
\hline S. enterica subsp. salamae $38: \mathrm{b}: 1,2$ & 40 & 40 & 50 & 17 & 26 & 28 & 30 & 34 & 50 \\
\hline S.I. $6,14,25:-: 1,2$ & 30 & 42 & 50 & 14 & 30 & 50 & 40 & 50 & 50 \\
\hline S. enterica subsp. houtenae 40:g,t:- & 30 & 44 & 46 & 12 & 15 & 25 & 30 & 44 & 60 \\
\hline Rough & 25 & 40 & 50 & $\mathrm{R}$ & $\mathrm{R}$ & $\mathrm{R}$ & 40 & 40 & 62 \\
\hline Rough & 29 & 36 & 40 & 12 & $30 *$ & $40 *$ & 40 & 40 & 56 \\
\hline Rough & 29 & 30 & 34 & $\mathrm{R}$ & $\mathrm{R}$ & 10 & 20 & 22 & 30 \\
\hline Rough & 30 & 35 & 42 & 10 & $25 *$ & $38 *$ & 40 & 40 & 58 \\
\hline
\end{tabular}


Table 3. Statistical analysis of Zone of Inhibition (ZOI) and the tested concentrations of 5-15, 5-30 and 15-30 $\mu 1$ for the antimicrobial activity of oregano, rosemary and thyme oils against Salmonella spp. Isolates.

\begin{tabular}{|c|c|c|c|c|c|c|c|c|c|}
\hline \multirow[b]{2}{*}{ Inhibitory activity } & \multicolumn{3}{|c|}{ OREGANO } & \multicolumn{3}{|c|}{ ROSEMARY } & \multicolumn{3}{|c|}{ THYME } \\
\hline & $5 \mu \mathrm{I}$ & $15 \mu \mathrm{l}$ & $30 \mu \mathrm{l}$ & $5 \mu \mathrm{I}$ & $15 \mu \mathrm{l}$ & $30 \mu \mathrm{l}$ & $5 \mu \mathrm{l}$ & $15 \mu \mathrm{l}$ & $30 \mu \mathrm{l}$ \\
\hline No Inhibition & - & - & - & 53 & 46 & 42 & - & - & - \\
\hline$<12 \mathrm{~mm}$ : weak activity zone & - & - & - & 54 & 52 & 48 & 1 & - & - \\
\hline $12 \mathrm{~mm} \geq$ inhibition zone $<20 \mathrm{~mm}$ : intermediate activity & - & - & - & 5 & 2 & 5 & 2 & 1 & - \\
\hline$\geq 20 \mathrm{~mm}$ : strong activity & 59 & 59 & 59 & - & 5 & 6 & 56 & 58 & 59 \\
\hline Range Zone of Inhibition (mm)n ZOI & $20-40$ & $23-50$ & $30-60$ & $10-17$ & $10-30$ & $10-50$ & $11-42$ & $12-50$ & $30-70$ \\
\hline Mean Zone of Inhibition (mm) & 27.36 & 34.90 & 41.29 & 1.32 & 3.75 & 5.59 & 28.42 & 36,69 & 47,29 \\
\hline Mode & 30 & 30 & 35 & 0 & 0 & 0 & 20 & 40 & 50 \\
\hline Std. Deviation & 4,926 & 5,818 & 6,457 & 4,023 & 8,014 & 11,264 & 8,770 & 8,756 & 10,830 \\
\hline
\end{tabular}

in pig production in Greece and across Europe [62]. Specifically, their use as growth promoters negatively affects the typical gut microflora, allowing the preferential colonization of the gut by organisms such as Salmonella spp. This colonization is making pigs an important risk for the contamination of pork carcasses and eventually pork products [63]. Among Salmonella serovars the monophasic $S$. Typhimurium has exhibited the highest antimicrobial resistance across Europe and in Greece $[4,64,65]$.

WHO reports Salmonella spp. as one of the most prevalent bacterial species causing food-borne disease and a major health threat due to fast developing antimicrobial resistance [61]. The spreading of such resistant strains hampers the therapeutic possibilities of human Salmonella infections increasing the need of alternative treatments replacing commonly used antimicrobials in animal production. Thus, an increasing number of researchers give evidence of the EOs' potential inhibitory activity against pathogens, such as Salmonella spp. $[18,27]$, reporting them as 'Generally Regarded As Safe' (GRAS) for both animals and humans, because they do not promote antimicrobial resistance, as observed with the long-term use of synthetic antibiotics $[66,67]$. In the present study, the evaluated EOs showed varying degrees of bactericidal ability against the tested Salmonella isolates at different treatment amounts. Rosemary was found less potential compared to oregano and thyme. Oregano and thyme showed high inhibitory effect, even for the very small amount of $5 \mu \mathrm{l}$ (Table 2 and 3 ) against all the salmonellae tested. An example is thyme oil, which at the amount of $30 \mu \mathrm{l}$ produced the largest $\mathrm{ZOI}$ in the study $(70 \mathrm{~mm})$ and showed the greatest mean ZOI $(47.29 \mathrm{~mm})$.

The obtained results showed that the inhibitory effect was dependent on the amount of the EO employed, showing that ZOI differed significantly only for the amount of $30 \mu \mathrm{l}$, with regards to the EO employed. This is of minor importance, because both oregano and thyme showed strong activity $(>20 \mathrm{~mm})$ for this particular amount. The Salmonella serovar appearing dependent on the EO used, was the monophasic variant of $S$. Typhimurium, S. enterica subsp. enterica ser. 4,12:i:-. This serovar was more sensitive against oregano and thyme EOs (Table 2, Figures 1-3). A comparison of the antibacterial activity of these EOs with that of commonly used antimicrobials, showed that the EOs have a better killing ability against Salmonella isolates, independent of the level of resistance to commercially used antibiotics, an observation similar to that reported by Mayaud et al. [68]. The present findings, although in vitro, suggest that the tested EOs rich in thymol and carvacrol could control a wide range of microbes, becoming potential alternatives to commonly used antimicrobials. However, their effectiveness in animals, when administrated orally, must be experimentally demonstrated in vivo as to the maximum amount of EO administrated for avoiding toxicity or affecting the gut microflora.
Table 4. Statistical analysis of Zone of Inhibition (ZOI) and the tested concentrations of 5-15, 5-30 and 15-30 $\mu \mathrm{l}$ for the antimicrobial activity of oregano, rosemary and thyme oils against Salmonella spp. pig isolates.

\begin{tabular}{|c|c|c|c|c|}
\hline Quantity & Essential Oil & $\mathbf{t}$ & $\mathbf{d f}$ & $\mathbf{p}$ \\
\hline \multirow{3}{*}{$5-15 \mu \mathrm{l}$} & oregano & -12.660 & 58 & $<0.001$ \\
\cline { 2 - 5 } & thyme & -9.389 & 58 & $<0.001$ \\
\cline { 2 - 5 } & rosemary & -3.791 & 58 & $<0.001$ \\
\hline \multirow{3}{*}{$5-30 \mu 1$} & oregano & -18.828 & 58 & $<0.001$ \\
\cline { 2 - 5 } & thyme & -8.685 & 58 & $<0.001$ \\
\cline { 2 - 5 } & rosemary & -4.201 & 58 & $<0.001$ \\
\hline \multirow{3}{*}{$15-30 \mu 1$} & oregano & -11.502 & 58 & $<0.001$ \\
\cline { 2 - 5 } & thyme & -10.385 & 58 & $<0.001$ \\
\cline { 2 - 5 } & rosemary & -3.397 & 58 & $<0.001$ \\
\hline
\end{tabular}

Table 5. Repeated Measures ANOVA comparing in pairs the antimicrobial activity of oregano, rosemary and thyme oils against Salmonella spp. isolates recovered from pigs

\begin{tabular}{|c|c|c|c|c|}
\hline \multirow{2}{*}{ quantity } & pairs & $\mathbf{t}$ & $\mathbf{d f}$ & $\mathbf{p}$ \\
\hline \multirow{3}{*}{$5 \mathrm{ml}$} & oregano - rosemary & 40,558 & 58 & $<0.001$ \\
\cline { 2 - 5 } & oregano - thyme & $-0,883$ & 58 & 0,381 \\
\cline { 2 - 5 } & rosemary- thyme & -24.612 & 58 & $<0.001$ \\
\hline \multirow{3}{*}{$15 \mathrm{ml}$} & oregano - rosemary & 27,006 & 58 & $<0.001$ \\
\cline { 2 - 5 } & oregano - thyme & $-1,414$ & 58 & 0,163 \\
\cline { 2 - 5 } & rosemary- thyme & $-22,642$ & 58 & $<0.001$ \\
\hline \multirow{3}{*}{$30 \mathrm{ml}$} & oregano - rosemary & 23,544 & 58 & $<0.001$ \\
\cline { 2 - 5 } & oregano - thyme & $-4,486$ & 58 & $<0.001$ \\
\cline { 2 - 5 } & rosemary- thyme & $-21,161$ & 58 & $<0.001$ \\
\hline
\end{tabular}

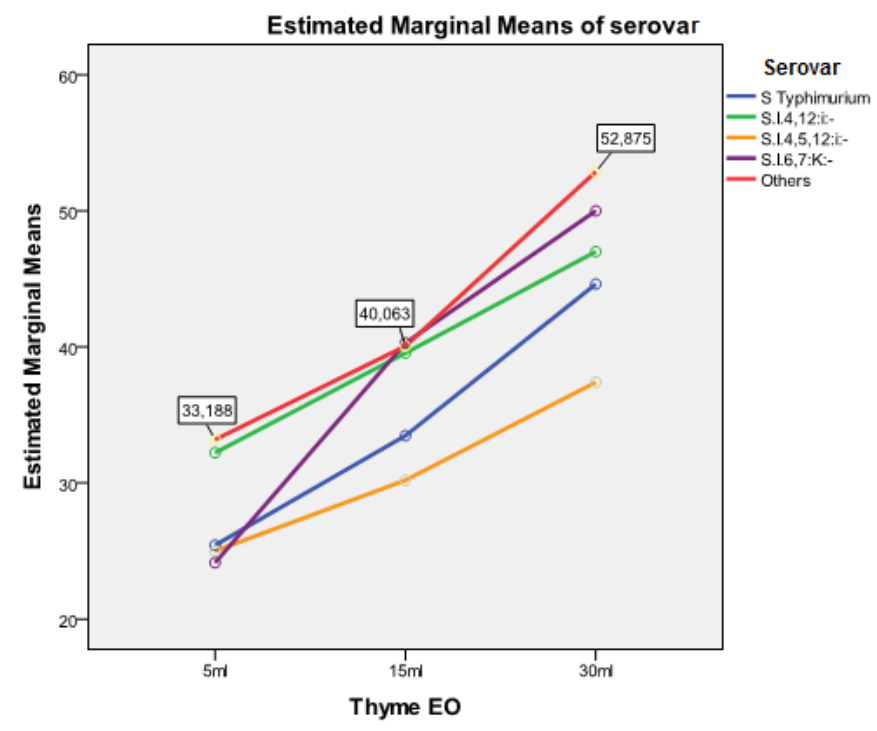

Figure 1. Estimated marginal means of Salmonella serovars with regards to thyme EO 


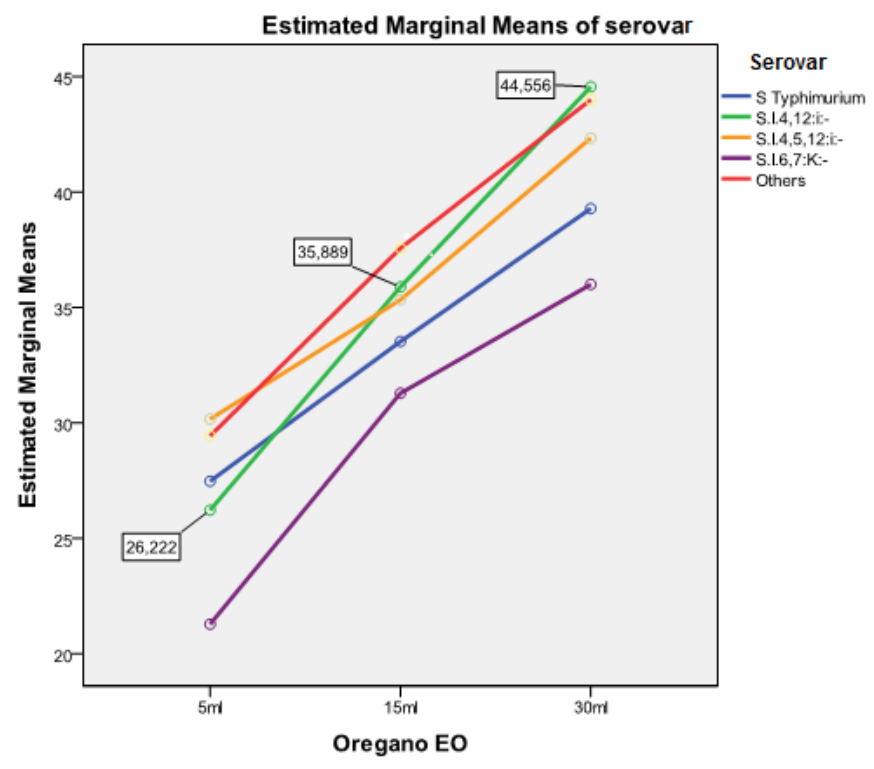

Figure 2. Estimated marginal means of Salmonella serovars with regards to oregano EO

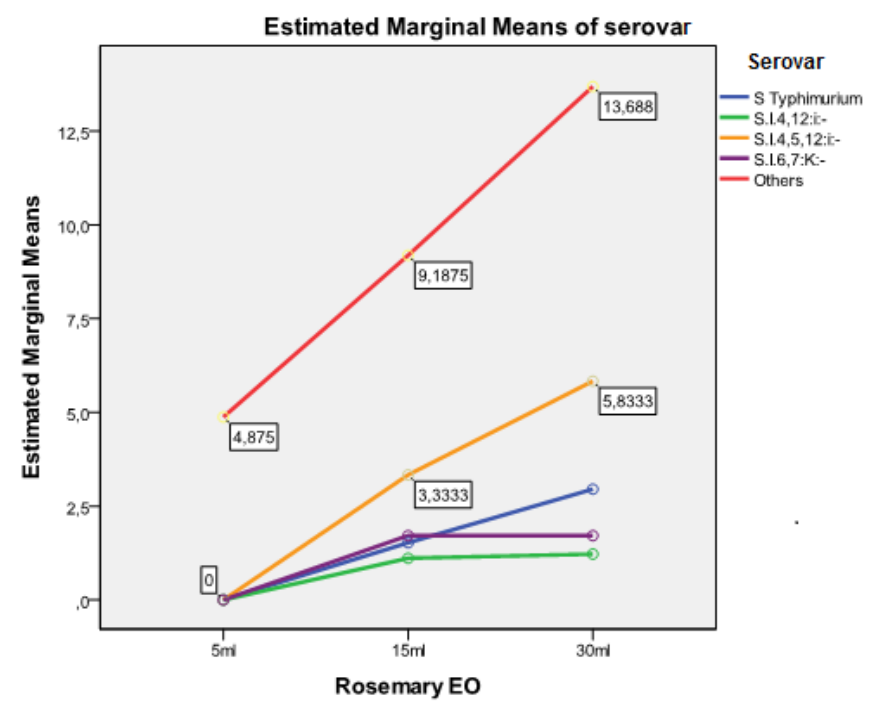

Figure 3. Estimated marginal means of Salmonella serovars with regards to rosemary EO.

In addition, a standardization of the methodology to evaluate their inhibitory activity should be established.

The unnecessary use of antibiotics has been a major promoter of the emergence and transmission of drug-resistant bacterial strains. Resistance to antimicrobials, whether attributed to animal or human excessive use, demonstrates the dynamic evolution of microorganisms' response to the hostile environment of antibiotics becoming a threat to human health. The food animal reservoir of such strains is an important source for human infections. By eliminating the use of drugs important for human medicine in animals, through the administration of EOs, the prevalence of resistant isolates could be reduced for the benefit of consumers.

In conclusion, EOs should be considered possible alternatives to synthetic drugs, administrated as growth promoters. The exploitation of
EOs as potential growth promoters in animals is the new hope of using phytopharmaceuticals against multidrug-resistant microorganisms. The present results add to the knowledge of previous studies, encouraging for more research into establishing EOs as reliable curative agents.

\section{References}

1. EFSA (European Food Safety Authority) (2010) Scientific Opinion on a Quantitative Microbiological Risk Assessment of Salmonella in slaughter and breeder pigs EFSA Panel on Biological Hazards (BIOHAZ). EFSA Journal 8: 1547.

2. FCC, Food Control Consultants Ltd Consortium (2010) Analysis of the costs and benefits of setting a target for the reduction of Salmonella in slaughter pigs for EUROPEAN COMMISSION Health and Consumers Directorate-General SANCO/2008/E2/036 FINAL REPORT. pp. 1-198.

3. Wedel SD, Bender JB, Leano FT, Boxrud DJ, Hedberg C, et al. (2005) Antimicrobialdrug susceptibility of human and animal Salmonella Typhimurium, Minnesota, 1997-2003. Emerg Infect Dis 11: 1899-1906. [Crossref]

4. EFSA (European Food Safety Authority), ECDC (European Centre for Disease Prevention and Control) (2014) The European Union Summary Report on antimicrobial resistance in zoonotic and indicator bacteria from humans, animals and food in 2012. EFSA Journal 12: 3590 .

5. Boyen F, Haesebrouck F, Maes D, Van Immerseel F, Ducatelle R, et al. (2008) Nontyphoidal Salmonella infections in pigs: A closer look at epidemiology, pathogenesis and control. Vet Microbiol 130: 1-19. [Crossref]

6. Helms M, Ethelberg S, Molbak K (2005) International Salmonella Typhimurium DT104 Infections, 1992-2001. Emerg Infect Dis 11: 859-867. [Crossref]

7. EFSA (European Food Safety Authority) (2009) Joint opinion on antimicrobial resistance (AMR) focused on zoonotic infections. EFSA Journal 7:1372.

8. van Duijkeren E, Wannet WJ, Houwers DJ, van Pelt W (2002) Serotype and phage type distribution of Salmonella strains isolated from humans, cattle, pigs, and chickens in the Netherlands from 1984 to 2001. J Clin Microbiol 40: 3980-3985. [Crossref]

9. Canton R, Morosini MI (2011) Emergence and spread of antibiotic resistance following exposure to antibiotics. FEMS MicrobiolRev 35: 977-991. [Crossref]

10. Mellon M, Benbrook C, Benbrook KL (2001) Hogging It!: Estimates of Antimicrobial Abuse in Livestock. Union of Concerned Scientists. UCS Publications, Cambridge, MA 02238-9105. Available from: http://www.ucsusa.org/assets/documents/food_and agriculture/hog_front.pdf.

11. Fair RJ, Tor Y (2014) Antibiotics and Bacterial Resistance in the 21st Century Perspect Medic Chem 6: 25-64. [Crossref]

12. Zaman SB, Hussain MA, Nye R, Mehta V, Mamun KT, et al. (2017) A Review on Antibiotic Resistance: Alarm Bells are Ringing. Cureus 9: e1403. [Crossref]

13. Joint FAO/OIE/WHO (2003) Expert Workshop on Non-Human Antimicrobial Usage and Antimicrobial Resistance: scientific assessment. Geneva: World Health Organization.

14. EFSA (European Food Safety Authority), ECDC (European Centre for Disease Prevention and Control) (2018) The European Union summary report on antimicrobial resistance in zoonotic and indicator bacteria from humans, animals and food in 2016 EFSA Journal 16: 5182.

15. Gebreyes WA, Thakur S, Morrow WEM (2006) Comparison of prevalence, antimicrobia resistance, and occurrence of multidrug-resistant Salmonella in antimicrobial-free and conventional pig production. J Food Protect 69: 743-748. [Crossref]

16. Butaye P, Michael GB, Schwarz S, Barrett TJ, Brisabois A, White DJ (2006) The clonal spread of multidrug-resistant non-typhi Salmonella serotypes. Microbes Infect 8: 1891 1897. [Crossref]

17. Dorman HJD, Deans SG (2000) Antimicrobial agents from plants: antibacterial activity of plant volatile oils. J Appl Microbiol 88: 308-316. [Crossref]

18. Cattelan MG, de Castilhos MBM, Sales PJP, Hoffmann FL (2013) Antibacterial activity of oregano essential oil against foodborne pathogens. Nutri Food Sci 43: 169-174.

19. Guenther E (1948) The Essential Oils. D. Van Nostrand, New York.

20. Nychas GJE (1995) Natural antimicrobials from plants. Blackie Academic and Professional, London, pp: 58-89.

21. Solomakos N, Govaris A, Koidis P, Botsoglou N (2008) The antimicrobial effect of thyme essential oil, nisin and their combination against Escherichia coli O157:H7 in minced beef during refrigerated storage. Meat Sci 80: 159-166. [Crossref] 
22. Tajkarimia MM, Ibrahima SA, Cliverb DO (2010) Antimicrobial herb and spice compounds in food. Food Control 21: 1199-1218.

23. Preuss H, Echard B, Dadgai A, Talpur N, Manohar V, et al. (2005) Effects of essential oils and monolaurin on Staphylococus aureus: in vitro and in vivo studies. Toxicol Mech Method 15: 279-285. [Crossref]

24. McMeekin TA, Hill C, Wagner M, Dahl A, Toss T (2010) Ecophysiology of foodborne pathogens: essential knowledge to improve food safety. Int J Food Microbiol 139: 64-78. [Crossref]

25. Gavanjia S, Mohammadi E, Larki B, Bakhtaric A (2014) Antimicrobial and cytotoxic evaluation of some herbal essential oils in comparison with common antibiotics in bioassay condition. Integr Med Res 3: 142-152. [Crossref]

26. Yap PS, Yiap BC, Ping HC, Lim SH (2014) Essential oils, a new horizon in combating bacterial antibiotic resistance. Open Microbiol J 8: 6-14. [Crossref]

27. Bento MHL, Ouwehand AC, Tiihonen K, Lahtinen S, Nurminen P, et al. (2013) Essential oils and their use in animal feeds for monogastric animals - Effects on feed quality, gut microbiota, growth performance and food safety: a review. VET MED CZECH 58: 449-458.

28. Pers Z (2016) Essential Oils for Healing. Lulu Press, Inc.

29. Fournomiti M, Kimbaris A, Mantzourani I, Plessas S, Theodoridou I, et al. (2015) Antimicrobial activity of essential oils of cultivated oregano (Origanum vulgare), sage (Salvia officinalis), and thyme (Thymus vulgaris) against clinical isolates of Escherichia coli, Klebsiella oxytoca, and Klebsiella pneumoniae. Microb Ecol Health Dis 26: 23289. [Crossref]

30. Kokkini S, Karousou R, Dardioti A, Krigas N, Lanaras T (1997) Autumn essential oils of Greek oregano. Phytochemistry 44: 8836

31. Aligiannis N, Kalpoutzakis E, Mitaku S, Chinou IB (2001) Composition and antimicrobial activity of the essential oils of two Origanum species. J Agric Food Chem 49: 4168-4170. [Crossref]

32. Baydar H, Sagdic O, Ozkan G, Karadogan T (2004) Antimicrobial activity and composition of essential oils from Origanum: Thymbra and Satureja species with commercial importance in Turkey. Food Control 15: 169-172.

33. Botelho MA, Nogueira NAP, Bastos GM, Fonseca SGC, Lemos TLG, et al. (2007) Antimicrobial activity of the essential oil from Lippia sidoides, carvacrol and thymol against oral pathogens. Braz J Med Biol Res 40: 349-356. [Crossref]

34. Magi G, Marini E, Facinelli B (2015) Antimicrobial activity of essential oils and carvacrol and synergy of carvacrol and erythromycin, against clinical, erythromycinresistant Group A Streptococci. Front Microbiol 6: 165. [Crossref]

35. Burt S (2004) Essential oils: their antibacterial properties and potential applications in foods a review. Intern J Food Microbiol 94: 22353. [Crossref]

36. Kulisic T, Radonic A, Katalinic V, Milos M (2004) Use of different methods for testing antioxidative activity of oregano essential oil. Food Chem 85: 63340.

37. Room A (1986) A Dictionary of True Etymologies. Taylor \& Francis. Pp: 150.

38. Jiang Y, WuN, Fu YJ, Wang W, Luo M, et al. (2011) Chemical composition and antimicrobial activity of the essential oil of Rosemary. Environ Toxicol Pharmacol 32: 63-68.

39. Nordqvist Z (2017) Rosemary: Health benefits, precautions, and drug interactions. Medical News Today. Available from: http://www.medicalnewstoday.com/ articles/266370.php

40. Bozin B, Mimica-Dukic N, Samojlik I, Jovin E (2007) Antimicrobial and antioxidant properties of rosemary and sage (Rosmarinus officinalis L. and Salvia officinalis L., Lamiaceae) essential oils.J Agric Food Chem 55: 7879-7885. [Crossref]

41. Habtemariam S (2016) The Therapeutic Potential of Rosemary (Rosmarinus officinalis) Diterpenes for Alzheimer's Disease. Evid Based Complement Alternat Med: 2680409. [Crossref]

42. Baranauskiene R, Venskutoni SPR, Viskelis P, Dambrauskiene E (2003) Influence of nitrogen fertilizers on the yield and composition of thyme (Thymus vulgaris). $J$ Agric Food Chem 51: 77518. [Crossref]

43. Feizi A, Nazeri M (2011) Thyme essential oils (thymus vulgaris) alleviate vaccination reactions in broiler chickens. Ann Biol Res 2: 464-468.

44. Marina S, Jasmina G, Petar DM, Dejan B, Leo JLD (2010) Antibacterial effects of the essential oils of commonly consumed medicinal herbs using an in vitro model. Molecules 15: 7532-7546. [Crossref]

45. Di Pasqua R, De Feo V, Villani F, Mauriello G (2005) In vitro antimicrobial activity of essential oils from Mediterranean Apiaceae, Verbenaceae and Lamiaceae against foodborne pathogens and spoilage bacteria. Ann Microbiol 55: 13943.
46. Centre for Agriculture and Bioscience International (2018) Rosmarinus officinalis (rosemary). Available from: https://www.cabi.org/isc/datasheet/47678

47. Exarchou V, Nenadis N, Tsimidou M, Gerothanassis IP, Troganis A, Boskou D (2002) Antioxidant activities and phenolic composition of extracts from Greek oregano, Greek sage, and summer savory. J Agric Food Chem 50: 52949. [Crossref]

48. Rai M, Paralijar P, Jogee P, Agarkar G, Ingle AP (2017) Synergistic antimicrobial of essential oils in combination with nanoparticles: emerging trends and future perspectives. Int J Pharm 519: 67-78.

49. Evangelopoulou G, Kritas S, Govaris A, Burriel AR (2014) Pork meat as a potential source of Salmonella enterica subsp. arizonae infection in humans. J Clin Microbiol 52: 741-744. [Crossref]

50. Evangelopoulou G, Filioussis G, Kritas S, Christodoulopoulos G, Triantafillou LA et al. (2014) Short Communication. Colonisation of pig gallbladders with Salmonella species important to public health. Vet Rec: 102822.

51. Evangelopoulou G, Filioussis G, Kritas S, Ioannidis A, Burriel AR (2018) Presence of emerging Salmonella spp. serovars in pig farms: a risk to public health. J Dairy Vet Anim Res 7: 103-107.

52. Skandamis P, Koutsoumanis K, Fasseas K, Nychas GJ E (2001) Inhibition of oregano essential oil and EDTA on Escherichia coli O157:H7. Ital J Food Sc 13: 65-75.

53. Rota MC, Herrera A, Martinez RM, Sotomayor JA, Jordan MJ (2008) Antimicrobial activity and chemical composition of Thymus vulgaris, Thymus zygis and Thymus hyemalis essential oils. Food Control 19: 681-687.

54. Marques de Sa JP (1999) Applied Statistics. ( ${ }^{\text {nd }}$ edn), Springer. pp: 187-188.

55. M. Hollander, Wolfe D (2007) Nonparametric Statistical Methods ( $2^{\text {nd }}$ edn), Springer. pp: 106-135.

56. Kennedy D (2013) Time to deal with antibiotics. Science 342: 777.

57. Choffnes ER, Relman DA, Olsen L, Hutton R, Mack A (2012) Improving Food Safety Through a One Health Approach Workshop Summary Institute of Medicine (US). Washington (DC): National Academies Press (US).

58. EFSA (European Food Safety Authority) (2011) The European Union Summary Report on antimicrobial resistance in zoonotic and indicator bacteria from humans, animals and food in the European Union in 2009. EFSA Journal 9: 2154

59. EFSA (European Food Safety Authority) (2015) EFSA (European Food Safety Authority) and ECDC (European Centre for Disease Prevention and Control), EU Summary Report on antimicrobial resistance in zoonotic and indicator bacteria from humans, animals and food in 2013. EFSA Journal 13: 4036.

60. EFSA (European Food Safety Authority) (2017) European Food Safety Authority European Centre for Disease Prevention and Control. The European Union summary report on antimicrobial resistance in zoonotic and indicator bacteria from humans, animals and food in 2015.

61. WHO (2011) WHO list of Critically Important Antimicrobials (CIA). World Health Organization (WHO), $3^{\text {rd }}$ Revision.

62. Burch D (2005) Problems of antibiotic resistance in pigs in the UK. In Pract. 27: 37-43.

63. Friendship RM, Mounchili A, McEwen S, Rajic A (2009) Critical review of on-farm intervention strategies against Salmonella. BPEX/ZNCP.

64. Mandilara G, Lambiri M, Polemis M, Passiotou M, Vatopoulos A (2013) Phenotypic and molecular characterization of multiresistant monophasic Salmonella Typhimurium (4, [5],12:i:-) in Greece, 2006-2011. Euro Surveill 18: 20496. [Crossref]

65. EFSA (European Food Safety Authority) (2016) The European Union summary report on antimicrobial resistance in zoonotic and indicator bacteria from humans, animals and food in 2014. European Food Safety Authority European Centre for Disease Prevention and Control.

66. Kabara JJ (1991) Phenols and chelators. Blackie, London, pp: 200-214

67. Hogberg LD, Heddini A, Cars O (2010) The global need for effective antibiotics challenges and recent advances. Trends Pharmacol Sci 31: 509-515. [Crossref]

68. Mayaud L, Carricajo A, Zhiri A, Aubert G (2008) Comparison of bacteriostatic and bactericidal activity of 13 essential oils against strains with varying sensitivity to antibiotics. Lett Appl Microbiol 47: 167-173. [Crossref]

Copyright: (C2019 Evangelopoulou G. This is an open-access article distributed under the terms of the Creative Commons Attribution License, which permits unrestricted use, distribution, and reproduction in any medium, provided the original author and source are credited. 